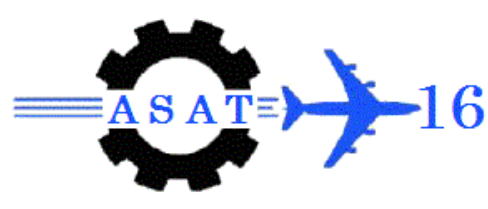

\title{
Design of Optimum Filament Wound Pressure Vessel with Integrated End Domes
}

\author{
Mahdy W. M. ${ }^{*}$, Kamel H. ${ }^{\dagger}$, and El-Soaly E.E. ${ }^{\ddagger}$
}

\begin{abstract}
In its most general form, a pressure vessel consists of two main parts; the cylindrical part and the dome part. This paper presents the analysis that has been conducted in order to obtain the optimum shape of the filament wound dome part. A composite pressure vessel with optimized dome ends avoids critical stresses that are incorporate with the structure when the structure is internally pressurized. The analysis deals with domes with/without polar opening. Analytical models are developed based on mechanics of materials, geodesic analysis and are solved using numerical techniques. The models can predict both the optimum shape and optimum thickness of the dome part that can safely withstand the applied loads with minimum weight. The results have been verified using published work with good agreement. Finally, the models have used to investigate the effect of changing material type, material properties on the optimum shape of the dome part.
\end{abstract}

\section{Keywords}

Filament wound pressure vessels, Geodesic-dome profile, optimum composite

\section{Introduction}

A pressure vessel is a closed container designed to hold gases or liquids at a pressure substantially different from the ambient one. In its most general form, a pressure vessel consists of two main parts; the cylindrical part and the dome part. New possibilities to improve the performance of pressure vessels were offered in the middle of the $20^{\text {th }}$ century and were associated with the development of filament winding technology. Filament winding is a type of composite manufacturing process where controlled amount of resin and oriented fibers are wounded around a rotating mandrel and are cured to produce the required composite part. Its typical application include the manufacture of: pressure vessels, rocket motor cases, aircraft fuselages, wing sections, radar domes, helicopter rotor shafts, highpressure pipelines, and sports goods [1].

The dome part experiences high stress values hence it is more susceptible to failure than the cylindrical part, which emphasizes the importance of its design. An optimum design is the one that guarantees different contradicting requirements; a high burst pressure and a large internal volume at the lowest possible weight [2]. This makes the design of optimum pressure vessels a considerable complex design problem that many researches have tried to solve; a brief summary of pertinent research is presented in the next section.

\footnotetext{
*MSc, Egyptian Armed Forces

${ }^{\dagger}$ Egyptian Armed Forces

* Professor, High Institute of Technology of $10^{\text {th }}$ Ramadan, October branch, Egypt
} 
Fukunaga et al. [3] presented two methods for determining the optimum shapes of filamentwound domes. One is based on the failure criteria of composite materials and the other is based on the performance factor. Liang, C.C. et al. [4] investigated the optimum design of dome contours for filament-wound pressure vessels. Geometrical limitations, winding condition, Tsai-Wu failure criterion, and maximizing shape factor were taken into account in design procedures. $\mathrm{Zu}$, L. et al. [5] showed a design method for determining the optimal meridian shape and related structural efficiency for non-geodesically overwound domes. The continuum lamination theory and the equal shell strains condition were used to outline the optimal meridian shapes of non-geodesics-based domes for various material anisotropy parameters. The dome thickness varies as a function of dome radius and winding pattern, which complicates the prediction of the dome thickness. Vafaeesefat, A. [2] introduced an algorithm for shape optimization of composite pressure vessel domes Based on rational Bspline curve and genetic algorithm. The genetic algorithm was used due to its great versatility, easy implementation and its ability for locating the globally optimal solution. Madhavi, M. et al. [6, 7] used netting analyses to calculate hoop and helical thicknesses for both cylinder and end domes. The distribution of winding angles through the dome part is calculated using Claurit's principle. The hoop components of the helical layers are not sufficient to fulfill the hoop reinforcement requirement, hence hoop winding is required. However, it is not possible to wind hoop layers on the end domes directly by filament winding technique. Therefore, an additional layer (called a doily) is placed as reinforcement on the end dome. This layer is in the form of a unidirectional fabric or drum wound hoop layers. Kumar, S. et al. [8] used geodesic path equation in order to predict the optimum meridian of filament-wound dome. The thicknesses of laminate structure at various portions of the dome and cylindrical regions are calculated by using netting analysis. A new method for predicting the dome thickness of composite pressure vessels based on cubic spline function was established by Wang, R. [9].

The main objective of this paper is to develop mathematical models for finding the shape and thickness of the optimum dome the one that can safely contains maximum volume with minimum weight. In the following section the governing equations are explained followed by description of the models development process. The models are solved numerically using MATLAB and the results are compared with previously published work. The models are then used to investigate the effect of material properties on optimum shape and thickness.

\section{Models Development}

\subsection{Governing equations}

Consider a filament-wound membrane shell of revolution. The shell is loaded with uniform internal pressure $\mathrm{p}$ and axial forces $\mathrm{T}$ uniformly distributed along the contour of the shell cross section $r=r_{0}$ as shown in Figure (1). Meridian, $\sigma_{\alpha}$, and circumferential, $\sigma_{\beta}$, stress acting in the shell follow from the corresponding free body diagrams of the shell element and can be written as [10]

$$
\begin{aligned}
& \sigma_{\alpha}=-Z \frac{\lambda}{r z^{\prime} h} \\
& \sigma_{\beta}=-\frac{\lambda}{z^{\prime} h}\left(p r-\frac{Z z^{\prime \prime}}{\lambda z^{\prime}}\right)
\end{aligned}
$$

where $\mathrm{z}(\mathrm{r})$ specifies the form of the shell meridian, $z^{\prime}=\frac{d z}{d r}$ and 


$$
\begin{aligned}
& Z=T r_{0}+\frac{p}{2}\left(r^{2}-r_{0}^{2}\right) \\
& \lambda=\sqrt{1+\left(z^{\prime}\right)^{2}}
\end{aligned}
$$

Meridian and circumferential stresses can be expressed in terms of stresses in the principal material coordinates of the tape, in case of orthotropic tape winding at $\pm \phi$ as:

$$
\begin{aligned}
& \sigma_{\alpha}=\sigma_{1} \cos ^{2} \phi+\sigma_{2} \sin ^{2} \phi-\tau_{12} \sin 2 \phi \\
& \sigma_{\beta}=\sigma_{1} \sin ^{2} \phi+\sigma_{2} \cos ^{2} \phi+\tau_{12} \sin 2 \phi
\end{aligned}
$$

where $\mathrm{h}$ is the shell thickness. The strains in the principal material coordinates can be expressed in terms of meridian and circumferential as:

$$
\begin{aligned}
& \varepsilon_{1}=\varepsilon_{\alpha} \cos ^{2} \phi+\varepsilon_{\beta} \sin ^{2} \phi \\
& \varepsilon_{2}=\varepsilon_{\alpha} \sin ^{2} \phi+\varepsilon_{\beta} \cos ^{2} \phi \\
& \gamma_{12}=\left(\varepsilon_{\beta}-\varepsilon_{\alpha}\right) \sin 2 \phi
\end{aligned}
$$

Since the right-hand parts of equation (4) include only two strains $\varepsilon_{\alpha}$ and $\varepsilon_{\beta}$, hence a compatibility equation linking the strains in the principal material coordinates is needed. This equation is

$$
\left(\varepsilon_{1}-\varepsilon_{2}\right) \sin 2 \phi+\gamma_{12} \cos 2 \phi=0
$$

Substituting the stains $\varepsilon_{1}$ and $\varepsilon_{2}$ in terms of stress $\sigma_{1}$ and $\sigma_{2}$ into equation (5), we have

$$
\left[\frac{\sigma_{1}}{E_{1}}\left(1+v_{12}\right)-\frac{\sigma_{2}}{E_{2}}\left(1+v_{21}\right)\right] \sin 2 \phi+\frac{\tau_{12}}{G_{12}} \cos 2 \phi=0
$$

where $\mathrm{E}_{1,2}$ are Young's modulus in directions 1 and $2 ; v_{12,21}$ are Poisson's ratios in the 1-2 and 2-1planes.

By combining the previous equations, the stresses acting on the optimal shell have the following expression

$$
\sigma_{1,2}=\frac{\sigma_{\alpha} e_{1,2}}{e_{1} \cos ^{2} \phi+e_{2} \sin ^{2} \phi}, \quad \tau_{12}=0
$$

where $e_{1}=\frac{E_{1}}{1+v_{21}}, \quad e_{2}=\frac{E_{2}}{1+v_{12}}$

Substituting these stresses into strain-stress relations for plane stress, the strain in the optimal shell has the following form

$$
\varepsilon_{\alpha}=\varepsilon_{\beta}=\frac{\sigma_{\alpha}\left(1-v_{12} v_{21}\right)}{\left(1+v_{12}\right)\left(1+v_{21}\right)\left(e_{1} \cos ^{2} \phi+e_{2} \sin ^{2} \phi\right)}
$$

\subsection{Design of Geodesic Dome Profile}

The geodesic profile is the elliptical curve connecting the two points taking the consideration of shortest distance. We need to define geodesic profile between pole opening radius and cylindrical portion radius $[11,12]$.

By combining equations (1), (3) and (6); the equation of the shell meridian in the explicit form becomes

$$
\frac{r z^{\prime \prime}}{\lambda^{2} z^{\prime}}=\frac{p r^{2}}{Z}-\Phi
$$


where

$$
\Phi=\frac{\sin ^{2} \phi+k \cos ^{2} \phi}{\cos ^{2} \phi+k \sin ^{2} \phi}
$$

The " $\mathrm{k}$ " parameter is function of material properties and defined as:

$$
k=\frac{E_{2}\left(1+v_{21}\right)}{E_{1}\left(1+v_{12}\right)}
$$

The vessel meridian can be decomposed into two segments as shown in Figure (2). For the first part of the meridian where $R \geq r \geq r_{f}$, we should take $T=\frac{p r_{0}}{2}$ into equation (2) and the shell meridian (equation (9)) becomes

$$
\frac{r z_{1}^{\prime \prime}}{\lambda_{1}^{2} z_{1}^{\prime}}=2-\Phi
$$

where the subscript, 1, refers to the first part. For the second part, where $r_{f} \geq r \geq r_{0}$, we should take $T=0$ and $p=p_{f}$ in equation (2) and the shell meridian (equation (9)) becomes

$$
\frac{r z_{2}^{\prime \prime}}{\lambda_{2}^{2} z_{2}^{\prime}}=\frac{2 r^{2}}{r^{2}-r_{0}^{2}}-\Phi
$$

where the subscript, 2, refers to the second part. Equations (12) and (13) are solved numerically for the two parts, where they must satisfy the following boundary conditions:

$$
\begin{array}{ll}
\frac{1}{z_{1}^{\prime}(R)}=0, & z_{1}(R)=0, \\
z_{1}^{\prime}\left(r_{f}\right)=z_{2}^{\prime}\left(r_{f}\right), & z_{1}\left(r_{f}\right)=z_{2}\left(r_{f}\right)
\end{array}
$$

where $\frac{1}{z_{1}^{\prime}(R)}=0$ means that the tangent line to the shell meridian is parallel to the axis $\mathrm{z}$ at $r=R$. The conditions $z_{1}\left(r_{f}\right)=z_{2}\left(r_{f}\right)$ and $z_{1}^{\prime}\left(r_{f}\right)=z_{2}^{\prime}\left(r_{f}\right)$ must be achieved to ensure the continuity of the two meridian's segments at $r=r_{f}$.

\subsection{Optimum shell meridian without polar opening $(\mathrm{r} 0=0)$}

The two equations which specify the shell meridian are the same in case of $r_{0}=0$, hence only one of them is needed to solve. For geodesic winding of the shell with the polar hole of radius $r_{0}$, the geodesic path is defined as:

$$
r \sin \phi=r_{0}
$$

The exact solution of equation (12) cannot be found. Hence, the solution has been done numerically using trapezoidal numerical integration method (TNIM). Combining equations (10) and (15) at $r_{0}=0$ and then substituting in equation (12), we get

$$
\frac{r z^{\prime \prime}}{\lambda^{2} z^{\prime}}=2-k
$$

In the previous equation, the subscript is removed where the two parts of the shell meridian have the same slop. Transform equation (16) to first order differential equation, and then integrate it with $\left(\frac{1}{z^{\prime}(R)}=0\right)$ boundary condition given by equation (14). The first integration of equation (16) is 


$$
y=-\frac{\left(\frac{r}{R}\right)^{2-k}}{\sqrt{1-\left(\frac{r}{R}\right)^{4-2 k}}}
$$

where $y=z^{\prime}$, and the negative sign appears due to the slop of meridian is decreasing with increasing the radius. Equation (17) cannot be integrated exactly; hence the integration will be done numerically. Equation (17) can be simplified into the following form:

$$
\frac{z}{R}=-\int \frac{(x)^{2-k}}{\sqrt{1-(x)^{4-2 k}}} d x
$$

Integration of the above equation can be done by using TNIM as:

$$
z_{(x=1)}=z_{(x=0)}+\sum A_{i}
$$

where " $\mathrm{i}$ " is number of intervals in the specified domain. Combining equations (14) and (19), we get

$$
z_{(x=0)}=-\sum A_{i}=-\left(\sum A_{b}+A_{1}^{*}\right)
$$

where " $b$ " is the nearest point to the point $x=1$ as shown in Figure (3). In the domain $b \leq x \leq 1$, by using TNIM, the area under the curve has the following form

$$
A_{1}^{*}=\left(\frac{y(b)+y(1)}{2}\right)(1-x)
$$

Rearranging the above equation yields,

$$
A_{1}^{*}=\frac{h}{2} y(b)+\frac{1}{2} \frac{-(x)^{2-k}}{\sqrt{1-(x)^{4-2 k}}}(1-x)
$$

where " $\mathrm{h}$ " is the step. At $\mathrm{x}=1$; we find $A_{1}^{*}=\frac{0}{0}$, hence taking limit as follows

$$
A_{1}^{*}=\frac{h}{2} y(b)-\frac{\left[-(2-k) x^{1-k}+(3-k) x^{2-k}\right]}{(4-2 k) x^{3-2 k}} \sqrt{1-(x)^{4-2 k}}
$$

The second term in the previous equation is zero at $\mathrm{x}=1$ and any value of $\mathrm{k}$. By combining the above equation and equation (20), we get the equation that describes the meridian of optimum shell without polar opening as follows

$$
z_{(x=0)}=-\left(\sum A_{b}+\frac{h}{2} y(b)\right)
$$

\subsection{Optimum shell meridian with polar opening $\left(r_{0} \neq 0\right)$}

This case is more complicated than the previous one, where the shell meridian is divided into two segments. As previous derivation, the first integration of equation (12) yields

$$
y_{1}(x)=\frac{d z_{1}}{R}=-\frac{x^{3}\left[1+x_{0}^{2}(k-1)\right]^{\left(\frac{k+1}{2}\right)}}{\sqrt{\left[x^{2}+x_{0}^{2}(k-1)\right]^{(k+1)}-x^{6}\left[1+x_{0}^{2}(k-1)\right]^{(k+1)}}} d x
$$

where

$$
x_{0}=\frac{r_{0}}{R} \quad, \quad x_{f} \leq x \leq 1 \quad, \quad x_{f}=\frac{r_{f}}{R}
$$

Integration of (25) can be done using TNIM as follows 


$$
z_{(x=1)}=z_{\left(x=x_{f}\right)}+\sum A_{i}
$$

The above equation can be simplified considering equation (14) as

$$
z_{\left(x=x_{f}\right)}=-\sum A_{i}=-\left(\sum A_{f}+A_{2}^{*}\right)
$$

where

$$
A_{2}^{*}=\left(\frac{y_{1}(b)+y_{1}(1)}{2}\right)(1-x)
$$

Rearranging the above equation yields

$$
A_{2}^{*}=\frac{h}{2} y_{1}(b)+\frac{1}{2} \frac{-x^{3}\left[1+x_{0}^{2}(k-1)\right]^{\left.\frac{k+1}{2}\right)}}{\sqrt{\left[x^{2}+x_{0}^{2}(k-1)\right]^{(k+1)}-x^{6}\left[1+x_{0}^{2}(k-1)\right]^{(k+1)}}}(1-x)
$$

At $\mathrm{x}=1$; we find $A_{2}^{*}=\frac{0}{0}$, hence taking limit as follows

$$
A_{2}^{*}=\frac{h}{2} y_{1}(b)-\frac{\left(3 x^{2}-4 x^{3}\right)\left[1+x_{0}^{2}(k-1)\right]^{\left(\frac{k+1}{2}\right)} \sqrt{\left[x^{2}+x_{0}^{2}(k-1)\right]^{(k+1)}-x^{6}\left[1+x_{0}^{2}(k-1)\right]^{(k+1)}}}{2 x(k+1)\left[x^{2}+x_{0}^{2}(k-1)\right]^{k}-6 x^{5}\left[1+x_{0}^{2}(k-1)\right]^{(k+1)}}
$$

At $\mathrm{x}=1$, the second term in the previous equation is zero at $k \leq \frac{2-3 x_{0}^{2}}{1-3 x_{0}^{2}}$.

The above equation is simplified to the following form by considering that $k \leq 2$

$$
A_{2}^{*}=\frac{h}{2} y_{1}(b)
$$

By combining the above equation and equation (27), we have

$$
z_{\left(x=x_{f}\right)}=-\left(\sum A_{f}+\frac{h}{2} y_{1}(b)\right)
$$

Equation (30) describes the meridian of optimal shell with polar opening in the range $x_{f} \leq x \leq 1$. The meridian of optimal shell with polar opening in the range $0 \leq x \leq x_{f}$ can be obtained by double integration of equation (13). The first integration of equation (13) with aid of equation (14) yields

$$
y_{2}(x)=-\frac{x_{f}^{2}\left[1+x_{0}^{2}(k-1)\right]^{\left(\frac{k+1}{2}\right)}\left(x^{2}-x_{0}^{2}\right) x}{\sqrt{\left(x_{f}^{2}-x_{0}^{2}\right)^{2}\left[x^{2}+x_{0}^{2}(k-1)\right]^{(k+1)}-x_{f}^{4}\left[1+x_{0}^{2}(k-1)\right]^{(k+1)} x^{2}\left(x^{2}-x_{0}^{2}\right)^{2}}} d x
$$

The second integration of equation (31) describes the meridian of optimal shell with polar opening, and has been done by using TNIM.

\subsection{Optimum thickness of filament-wound dome}

Dome thickness is needed, in order to determine the behavior of composite pressure vessels. Prediction of the dome thickness is not easy, which it varies as a function of dome radius and winding pattern. A simple expression of the dome thickness is presented by [13], which follows from the condition of filament continuity:

$$
h(r)=h_{R} \frac{R \cos \varphi_{R}}{r \cos \varphi_{(r)}}
$$

where $R, \varphi_{R}$, and $h_{R}$ are radius, winding angle, and thickness at cylinder section; $\mathrm{r}, \varphi_{(r)}$, and $h$ are arbitrary shell radius, winding angle, and thickness at the vessel dome, respectively. Rearranging equation (32) yields 


$$
\frac{h(x)}{h_{R}}=\frac{\sqrt{1-x_{0}^{2}}}{\sqrt{x^{2}-x_{0}^{2}}}
$$

There are three expressions specify the dome thickness for the case of geodesic winding, which presented by [11]. For $1 \geq x \geq x_{f}$, the dome thickness is specified by the following expression

$$
\frac{h_{1}(x)}{h_{R}}=\frac{\lambda_{1} \sqrt{1-x_{0}^{2}}}{\bar{t}}\left[\sin ^{-1} \frac{x_{0}}{x}\left(1+\frac{\bar{t}}{\lambda_{1} x_{0}}\right)-\sin ^{-1} \frac{x_{0}}{x}\right]
$$

where $\bar{t}=\frac{t_{d}}{R}$, ' $\mathrm{t}_{\mathrm{d}}$ ' is the tape width. For $x_{f} \geq x \geq x_{0}+\bar{t}$, the dome thickness has the following expression

$$
\frac{h_{2}(x)}{h_{R}}=\frac{\lambda_{2} \sqrt{1-x_{0}^{2}}}{\bar{t}}\left[\sin ^{-1} \frac{x_{0}}{x}\left(1+\frac{\bar{t}}{\lambda_{2} x_{0}}\right)-\sin ^{-1} \frac{x_{0}}{x}\right]
$$

where

$$
\begin{aligned}
& \lambda_{1}=\sqrt{1+\left(z_{1}^{\prime}\right)^{2}} \\
& \lambda_{2}=\sqrt{1+\left(z_{2}^{\prime}\right)^{2}}
\end{aligned}
$$

For $x_{0} \leq x \leq x_{0}+\bar{t}$, the dome thickness has the following expression

$$
\frac{h_{3}(x)}{h_{R}}=\frac{\lambda_{2} \sqrt{1-x_{0}^{2}}}{\bar{t}} \cos ^{-1} \frac{x_{0}}{x}
$$

\section{Results and discussion}

The optimum meridian of filament-wound dome without polar opening is described in equation (24) as a function of material properties. In order to increase the accuracy of this numerical integration, a small step $\left(1 \times 10^{-5}\right)$ is used. Figure (4) shows the meridians of optimum shell for different material properties; these meridians are verified with the results of Vasiliev [13]. It can be shown that the results of the present approach are in very good agreement with the published results.

The optimum meridian of filament-wound dome with polar opening is found to be a function of material properties. In this paper, parameter $\mathrm{k}$ is used to describe material properties and the optimum meridians are calculated for a range of $\mathrm{k}$ from zero to one. The results are shown in Figure (5) and are verified with the ones of Vasiliev [13]. There is a good agreement with the published results in this range of ' $\mathrm{k}$ '. The ratio of polar opening radius to cylindrical radius is taken equal to 0.2 , while the ratio of flange radius to cylindrical radius is taken equal to 0.3. However, if ' $\mathrm{k}$ ' is larger than unity, the deviation between results from aforedeveloped models and published ones increases as shown in Figure (6). This difference is discussed in Table (1) by examining the slope of the two meridians. In case of shells without polar opening, the slope of two meridians is almost the same. For shells with polar opening, the slope of the meridian $\left(x_{f} \leq x \leq 1\right)$ of the published results is almost the same as the one shells without polar opening. This should not be the case and it is clear that the published results have been mistakenly typed.

The effect of type of composite material on the meridian of dome is shown in Figure (7). Three types of composite materials (see Table (2)) are used in this study. It is clear that the type of material has a strong effect on the optimum shape of the dome. To demonstrate the effect of changing specific longitudinal and transverse moduli of elasticity, Aramid/Epoxy is selected as the composite material for the case study. The results are shown in Figure (8) and 
Figure (9). It is interesting to note that longitudinal modulus has a higher effect on the optimum shape of the dome compared with the transverse modulus.

The dependence of the normalized thickness of filament-wound dome on the normalized radius from equation (33) is shown in Figure (10). As can be seen, the shell thickness increases while $x$ decreases from 1 to $x_{0}$. Fair prediction of shell thickness can be obtained only for a certain distance from the polar hole using equation(33). However the equation cannot be used in the close vicinity of the hole. Consider a shell made by geodesic winding with $\bar{t}=0.042$, dependence of the normalized thickness, which is specified by equations (34) : (36) on the shell radius is shown in Figure (11). By comparing Figure (10) and Figure (11), it can be observed that at a distance exceeding $2 \mathrm{t}$ from the polar hole, the two curves are identical. However, there is a sharp thickness peak near one-band width, as shown in Figure (11), which in reality may occur due to slipping, realignment and roving separation of the fiber tows, and material consolidation in the process of winding and curing. Due to the lack of analytical equations describing these effects, an approximate model is developed. This approximation has the following third-order polynomial:

$$
h_{a}=a_{0}+a_{1} r+a_{2} r^{2}+a_{3} r^{3}
$$

where $a_{0}, a_{1}, a_{2}$, and $a_{3}$ are unknown coefficients. The approximation is valid for $\left(r_{2 t}=r_{0}+2 t\right) \geq r \geq r_{0}$ and requires that:

- The dome thickness specified by equation (35) for $x_{f} \geq x \geq x_{2 t}$ and by equation (37) for $x_{0} \leq x \leq x_{2 t}$ is continuous and has continuous derivative at $x=x_{2 t}$, i.e.,

$$
h\left(x_{2 t}\right)=h_{a}\left(x_{2 t}\right), \quad h^{\prime}\left(x_{2 t}\right)=h_{a}^{\prime}\left(x_{2 t}\right)
$$

- Equations (34) : (37) provide the same material volume for $x_{0} \leq x \leq x_{2 t}$, i.e.,

$$
\int_{x_{0}}^{x_{2 t}} h \lambda_{2} x d x=\int_{x_{0}}^{x_{2 t}} h_{a} \lambda_{2} x d x
$$

The above conditions are enough to determine the unknown coefficients in equation(37).

The whole optimum pressure vessel can now be described where for the cylindrical section, $\mathrm{k}=2$ from equation(16). However, for vessel with domes, we must take $\mathrm{k}<2$; hence the cylindrical section should be additionally reinforced with fibers in the circumferential direction.

\section{Conclusion}

In this paper, a development of analytical models that describe optimum shape and thickness of the filament-wound dome with/without polar opening was presented. The developed models were verified by comparing them with published work. The verified models were used to investigate the effect of changing composite material type on the optimum shape of the dome. It was found that the longitudinal modulus has a higher effect on the optimum shape of the dome compared with the transverse modulus. The developed models can be used in the design of optimum pressure vessels and moreover in selection of most suitable composite materials. 


\section{Acknowledgement}

I am deeply indebted to Ass. Prof. Dr. Hamed Abdalla, and Dr. Mahmoud Yehya for their attention and supervising this research. They regard and guidance led to the completion of the work in the present form. Really, they don't spare any information for me.

\section{References}

[1] F. Abdalla, S. Mutasher, Y. Khalid, S. Sapuan, A. Hamouda, B. Sahari, et al., "Design and fabrication of low cost filament winding machine," Materials \& Design, vol. 28, pp. 234-239, 2007.

[2] A. Vafaeesefat, "Dome Shape Optimization of Composite Pressure Vessels Based on Rational B-Spline Curve and Genetic Algorithm," Applied Composite Materials, vol. 16, pp. 321-330, 2009.

[3] H. Fukunaga and M. Uemura, "Optimum design of helically wound composite pressure vessels," Composite structures, vol. 1, pp. 31-49, 1983.

[4] C. C. Liang, H. W. Chen, and C. H. Wang, "Optimum design of dome contour for filament-wound composite pressure vessels based on a shape factor," Composite structures, vol. 58, pp. 469-482, 2002.

[5] L. Zu, S. Koussios, and A. Beukers, "Design of filament-wound domes based on continuum theory and non-geodesic roving trajectories," Composites Part A: Applied Science and Manufacturing, vol. 41, pp. 1312-1320, 2010.

[6] M. Madhavi, "Design and Analysis of Filament Wound Composite Pressure Vessel with Integrated-end Domes," Defence Science Journal, vol. 59, pp. 73-81, 2009.

[7] M. Madhavi and K. Rap, "Computer Aided Analysis of Filament Wound Composite Pressure Vessel with Integrated End Domes considering the Change of Winding Angles through the Thickness Direction," Journal of the Institution of Engineers(India), Part MC, Mechanical Engineering Division, vol. 91, pp. 10-16, 2010.

[8] S. S. Kumar and A. S. Kumari, "Design And Failure Analysis Of Geodesic Dome Of A Composite Pressure Vessel," in International Journal of Engineering Research and Technology, 2012.

[9] R. Wang, W. Jiao, W. Liu, and F. Yang, "A new method for predicting dome thickness of composite pressure vessels," Journal of Reinforced Plastics and Composites, vol. 29, pp. 3345-3352, 2010.

[10] V. V. Vasiliev and E. V. Morozov, Advanced mechanics of composite materials, second edition ed.: Elsevier Science Limited, 2007.

[11] V. V. Vasiliev, Composite Pressure Vessels: Analysis, Design, and Manufacturing: Bull Ridge Corporation, 2009.

[12] M. Z. Kabir, "Finite element analysis of composite pressure vessels with a load sharing metallic liner," Composite structures, vol. 49, pp. 247-255, 2000.

[13] V. Vasiliev, A. Krikanov, and A. Razin, "New generation of filament-wound composite pressure vessels for commercial applications," Composite structures, vol. 62, pp. 449-459, 2003. 


\section{Appendix}

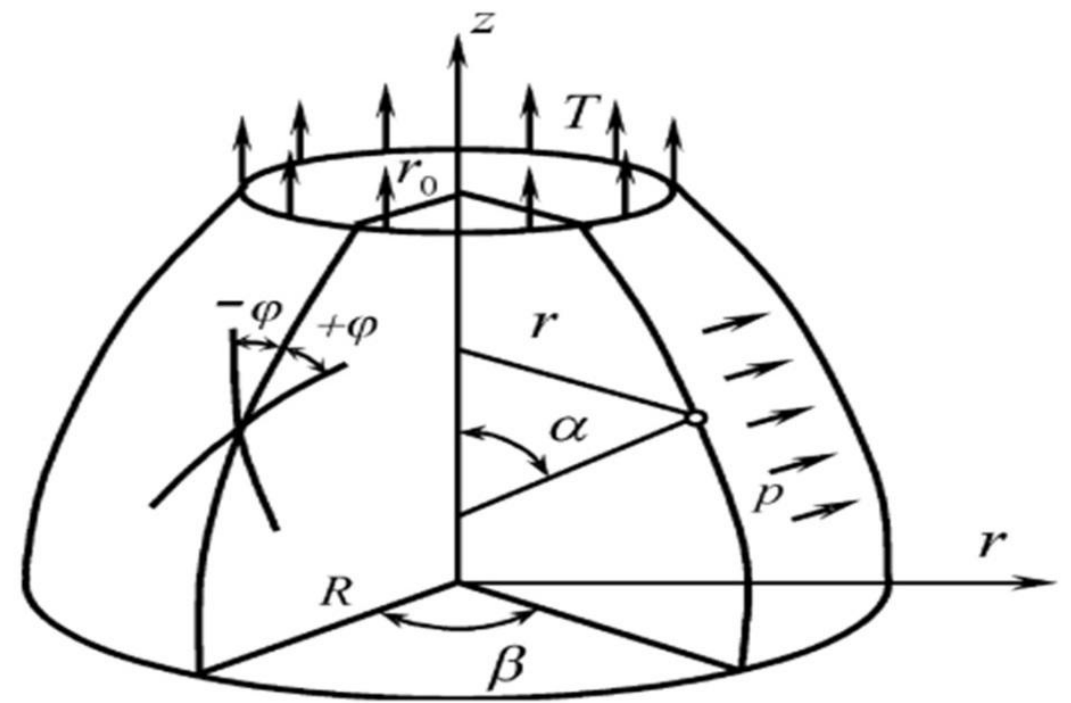

Figure (1) Axi-symmetrically loaded membrane shell of revolution [13]

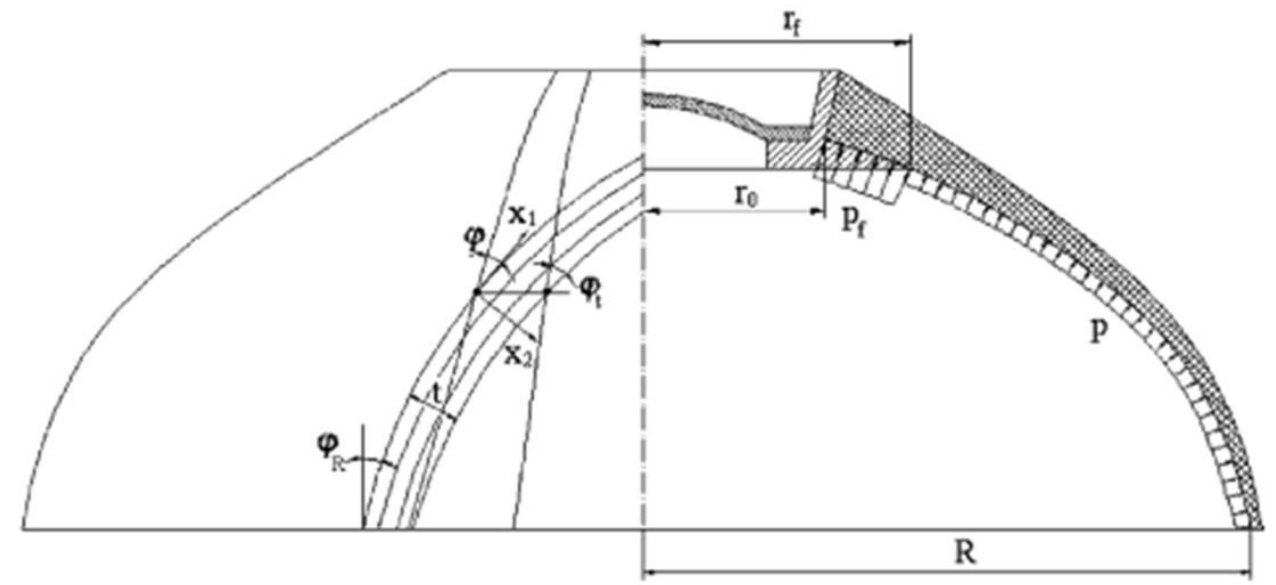

Figure (2) Structure of a composite pressure vessel dome [13]

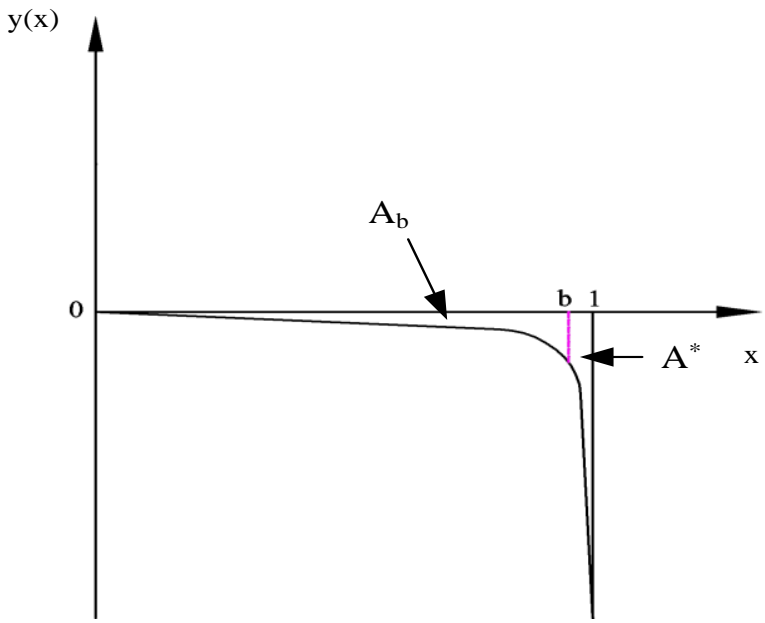

Figure (3) First derivative of meridian of optimal shell 


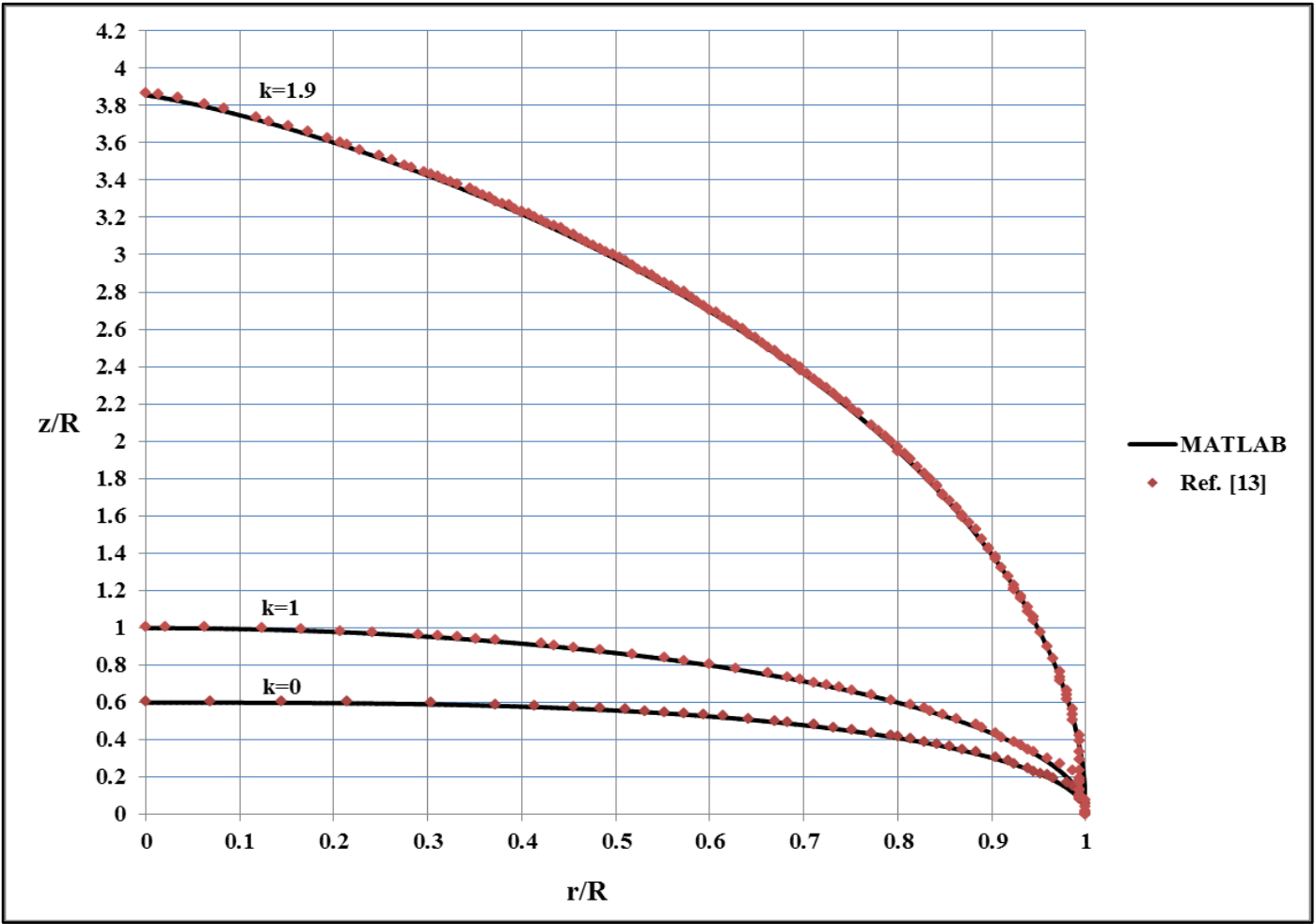

Figure (4) Meridian of optimum shells without polar opening

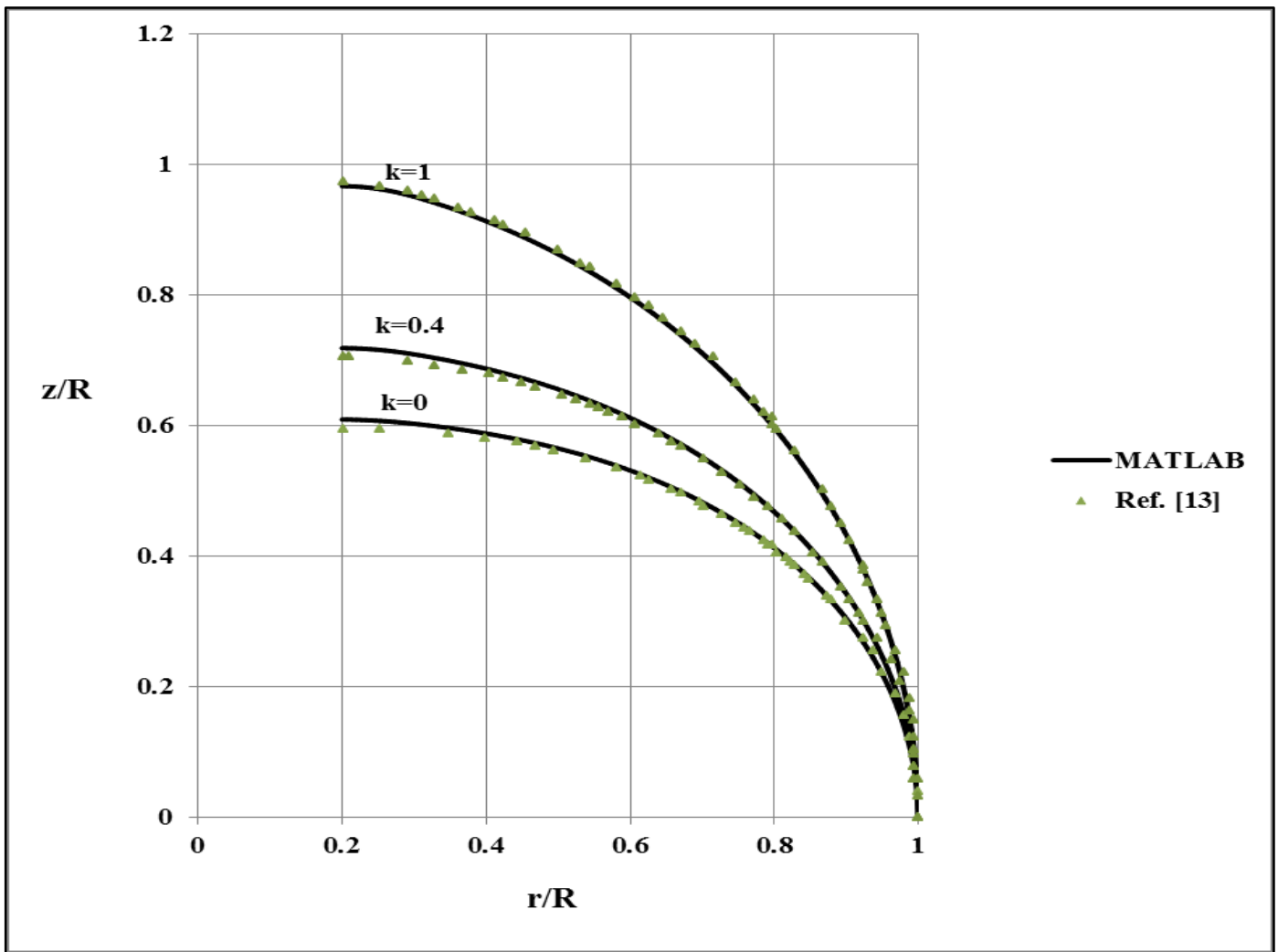

Figure (5) Meridians of optimum shell with polar opening (at $0 \leq k \leq 1)$ for $r_{0}=0.2 R$ 


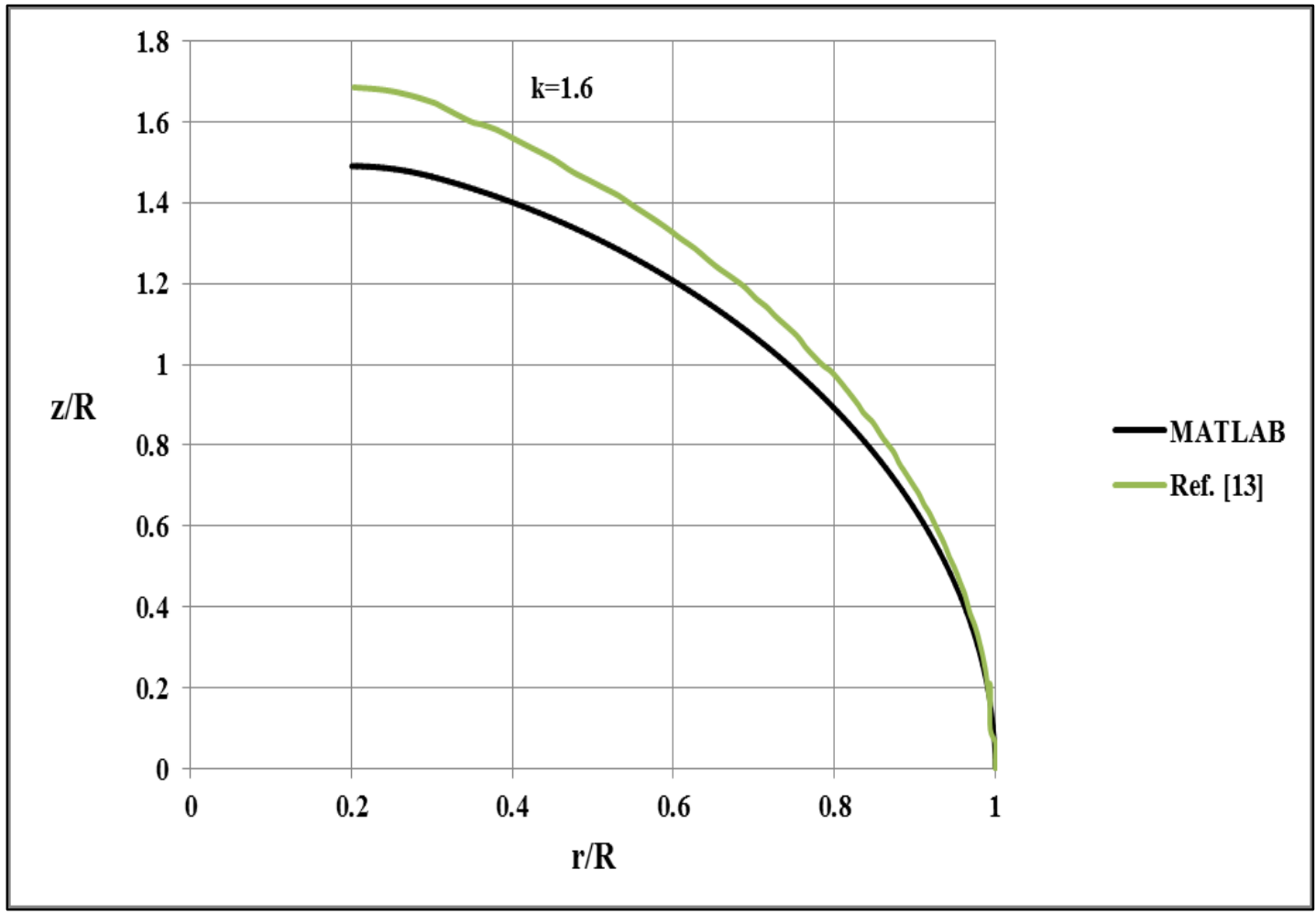

Figure (6) Comparison between the used technique and published results [13] for determining the meridian of optimum shell with polar opening for $k>1$

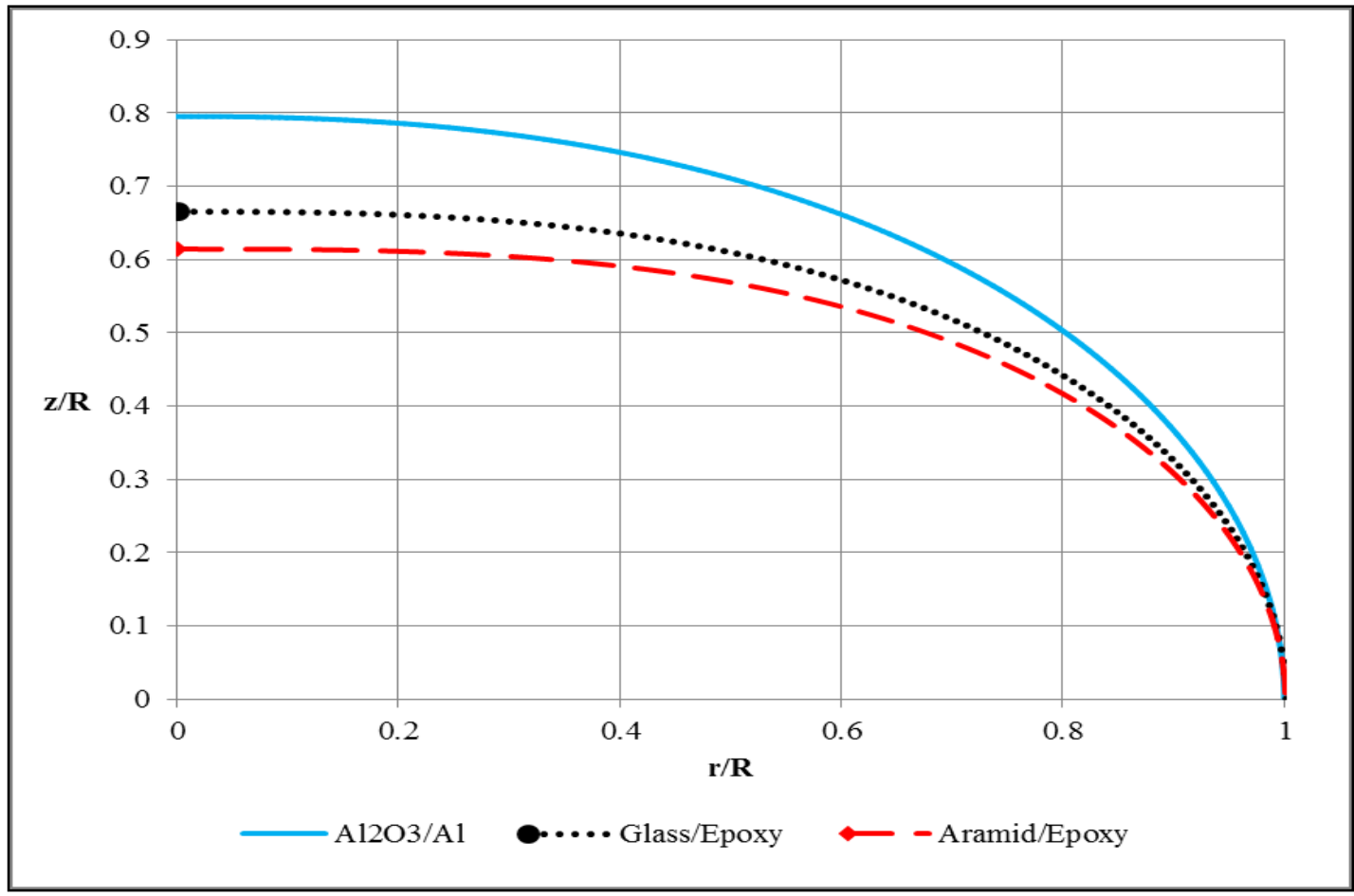

Figure (7) Effect of material properties on dome shape without polar opening 


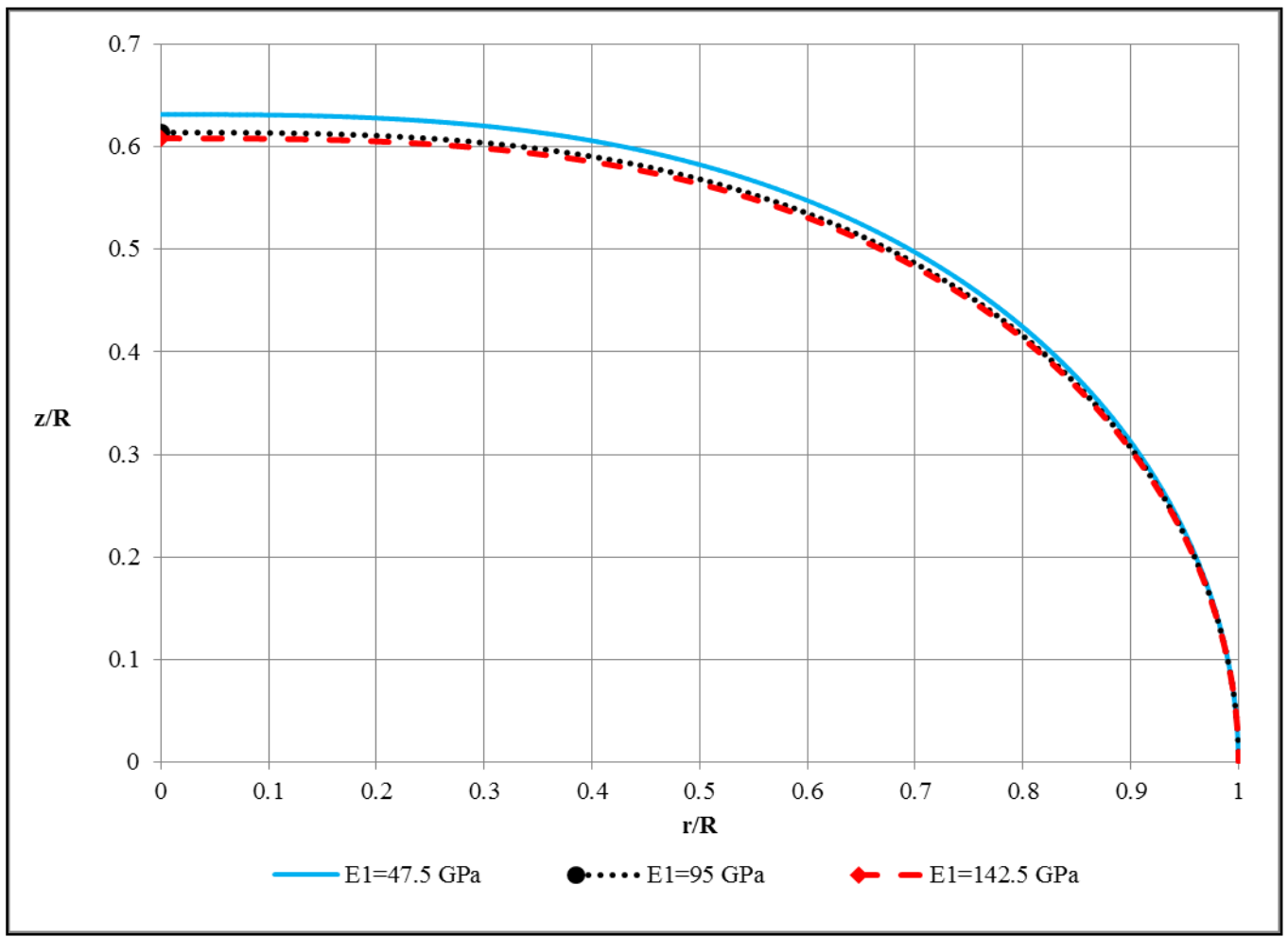

Figure (8) Effect of variation of longitudinal modulus $E_{1}$ of Aramid/Epoxy on dome shape

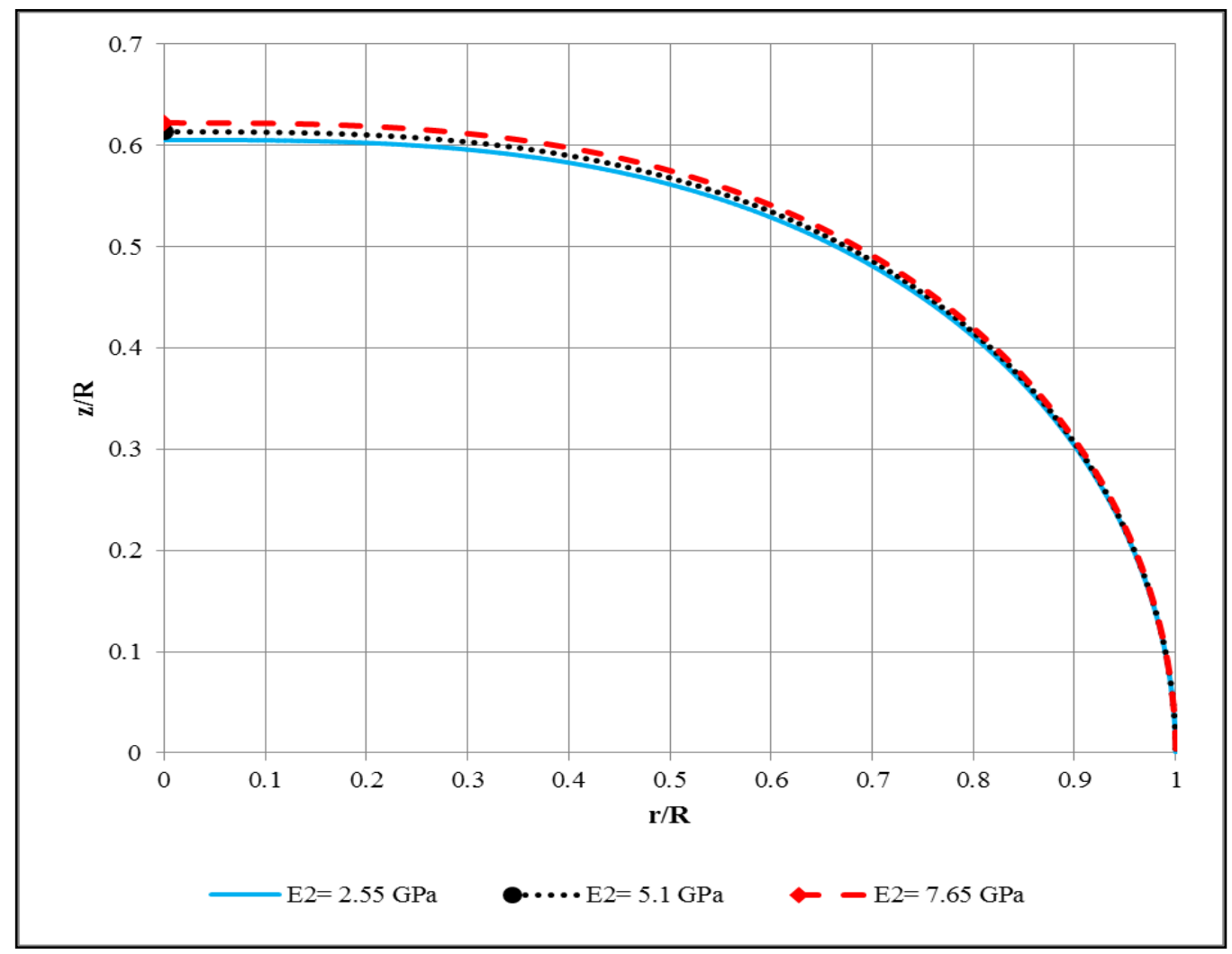

Figure (9) Effect of variation of transverse modulus $E_{2}$ of Aramid/Epoxy on dome shape 


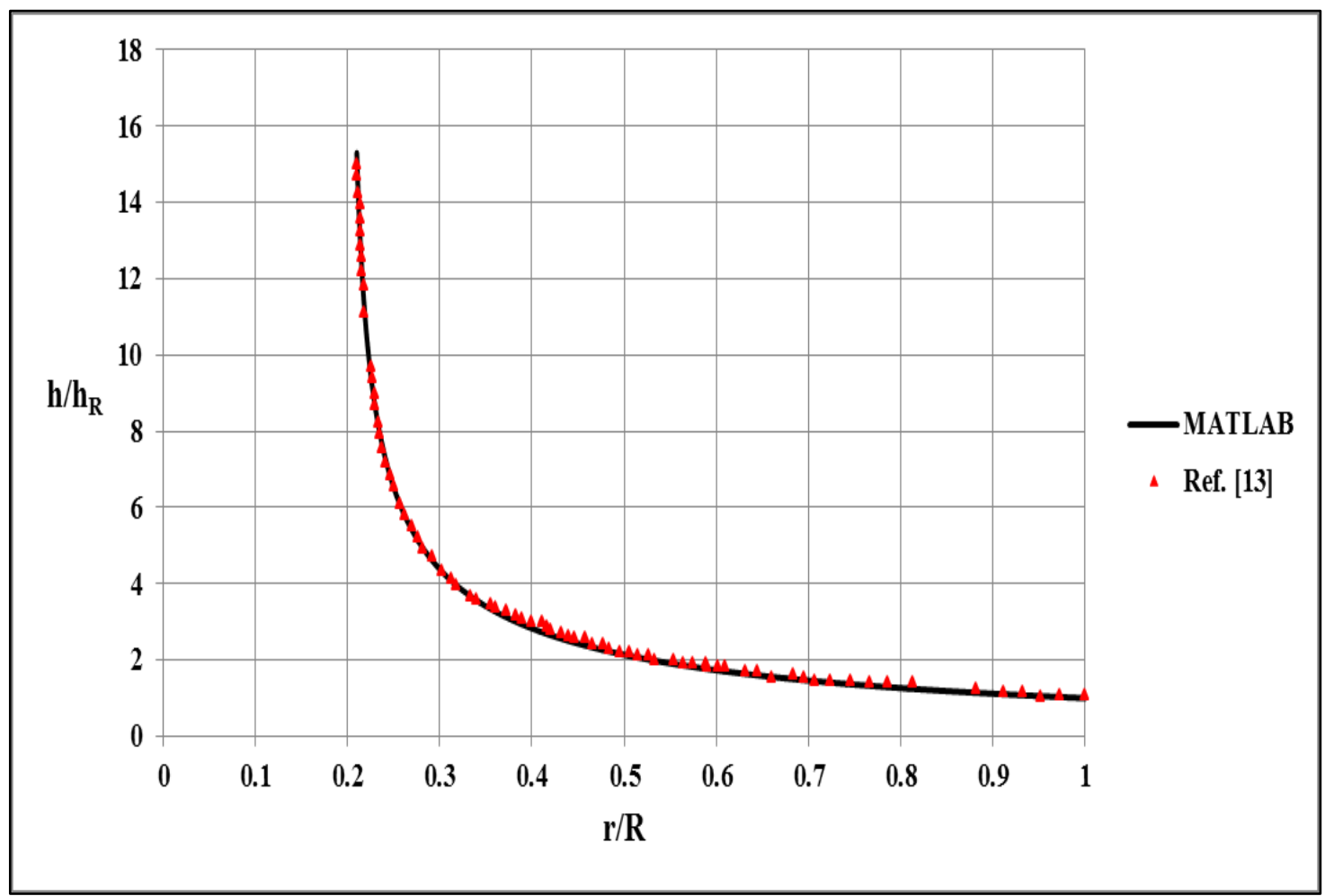

Figure (10) Dependence of the normalized thickness on radius, equation (33)

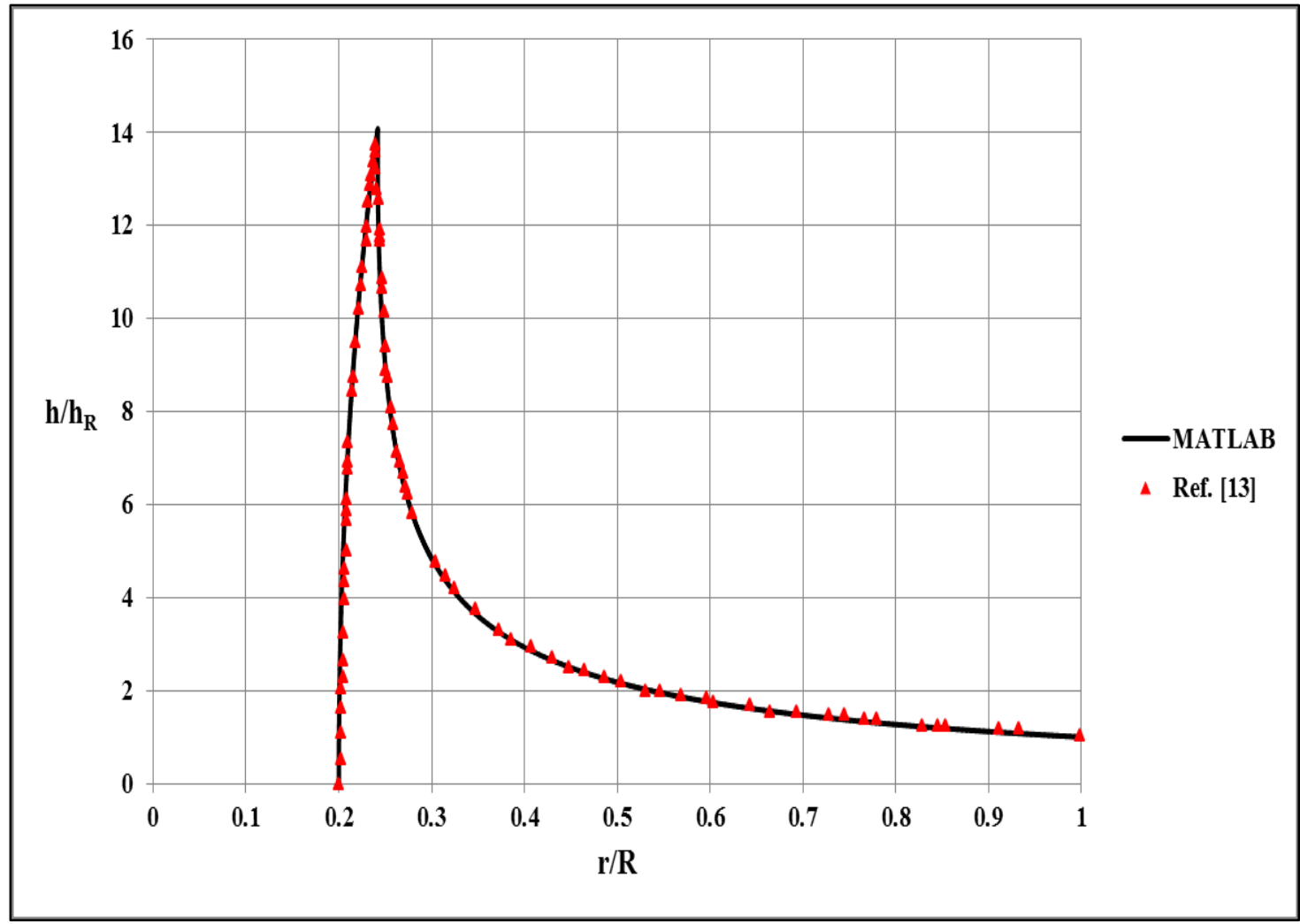

Figure (11) Dependence of the normalized thickness on radius from equations (34) : (36) 
Table (1) Comparison between the slope of optimal meridians from the used approach and the slope of optimal meridians from Ref. [13]

\begin{tabular}{|c|c|c|c|}
\hline \multirow{2}{*}{$\begin{array}{c}\text { Without polar } \\
\text { opening }\end{array}$} & $\begin{array}{c}\mathrm{k}=1.6 \\
\mathrm{y}(0.3)\end{array}$ & -0.785672 & Ref. [13] \\
\cline { 2 - 4 } & $\begin{array}{c}\mathrm{k}=1.8 \\
\mathrm{y}(0.5)\end{array}$ & -1.769128 & -0.79585 \\
\hline \multirow{4}{*}{ With polar opening } & $\begin{array}{c}\mathrm{k}=1.6 \\
\mathrm{y}(0.25)\end{array}$ & -0.259933 & -1.79935 \\
\cline { 2 - 5 } & $\begin{array}{c}\mathrm{k}=1.8 \\
\mathrm{y}(0.5)\end{array}$ & -1.201529 & -0.6747 \\
\hline
\end{tabular}

Table (2) Typical properties of some of composites [10]

\begin{tabular}{|c|c|c|c|}
\hline Property & $\begin{array}{c}\text { Aramid- } \\
\text { Epoxy }\end{array}$ & $\begin{array}{c}\text { Glass- } \\
\text { epoxy }\end{array}$ & $\begin{array}{c}\mathrm{Al}_{2} \mathrm{O}_{3-} \\
\mathrm{Al}\end{array}$ \\
\hline $\begin{array}{c}\text { Longitudinal } \\
\text { modulus, } \mathrm{E}_{1}(\mathrm{GPa})\end{array}$ & 95 & 60 & 260 \\
\hline $\begin{array}{c}\text { Transverse } \\
\text { modulus, } \mathrm{E}_{2}(\mathrm{GPa})\end{array}$ & 5.1 & 13 & 150 \\
\hline Poisson's ratio, $v_{21}$ & 0.34 & 0.3 & 0.24 \\
\hline
\end{tabular}

